\title{
Paediatric pancreatic trauma: A review of the literature and results of a multicentre survey on patient management
}

\author{
C Westgarth-Taylor, BSc, BSc (Med) Hons, MB ChB, MRCS (Eng), FC Paed Surg (SA); \\ J Loveland, MB ChB, FCS (SA), Cert Paed Surg \\ Department of Paediatric Surgery, Chris Hani Baragwanath Academic Hospital and Faculty of Health Sciences, \\ University of the Witwatersrand, Johannesburg, South Africa
}

Corresponding author: C Westgarth-Taylor(drcwtaylor@gmail.com)

\begin{abstract}
Background. It is generally accepted that paediatric solid organ injury should be treated conservatively, unless there is haemodynamic instability unresponsive to resuscitation. When it comes to pancreatic trauma, there is much debate about appropriate management.

Objectives. To review the literature and determine how pancreatic trauma is managed in South African (SA) tertiary institutions and compares with international standards.

Method. A survey was emailed to 45 paediatric surgical consultants working in various paediatric surgical units in SA, Italy, England and Australia. The questionnaire comprised two scenarios of isolated pancreatic trauma (grade III), the main difference between them being the time interval between initial injury and presentation. In the first scenario, the patient presented 6 hours post injury whereas in the second scenario, the patient presented 6 days post initial injury. The survey enquired about diagnosis and subsequent work-up (including preferred imaging techniques), supportive management (including nutrition), the various options of definitive intervention and follow-up procedure. Results. There were 21 responders from four different countries. In the first scenario, 10 surgeons would operate, 8 would treat conservatively and 3 would perform an endoscopic retrograde cholangiopancreatogram (ERCP) and stent. In the second scenario, 4 surgeons would operate, 13 would treat conservatively and 4 would undertake ERCP with stent. There was no difference in management between the SA surgeons and their international counterparts.

Conclusion. Management of blunt pancreatic trauma in SA is consistent with that reported in the literature. There is still controversy regarding the optimal management of pancreatic injury involving ducts. No absolute algorithm can be used to treat these patients. All patients should be treated individually and managed with an approach and techniques that are feasible.
\end{abstract}

S Afr Med J 2014;104(11):803-807. DOI:10.7196/SAMJ.8920

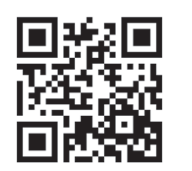

Trauma is one of the leading causes of morbidity and mortality in the paediatric population worldwide, with blunt abdominal trauma being the principal cause of abdominal injury in children. ${ }^{[1,2]}$ The pancreas is the fourth most common solid organ injured after the spleen, liver and kidneys. In the absence of haemodynamic instability unresponsive to resuscitation or associated hollow visceral injuries, non-operative management of these injuries is well documented and widely accepted by paediatric surgeons. However, the same cannot be said for blunt pancreatic injuries, as there is still much debate about the optimal management of affected patients. ${ }^{[3-6]}$

The pancreas is a retroperitoneal organ, draped over the bony vertebral column. Injury in children is usually due to compression against the underlying lumbar vertebrae, thus explaining the predilection for laceration or contusion at the pancreatic neck. Traumatic pancreatic injury is more common in children owing to their flatter diaphragm, thinner abdominal wall and higher costal margin. ${ }^{[7]}$ Despite these risk factors, blunt pancreatic trauma is relatively rare, with a reported incidence ranging from $0.3 \%$ to $0.7 \%$ of admissions for blunt abdominal trauma. ${ }^{[7,8]}$ This makes it difficult for individual centres to research management protocols owing to the limited number of cases, and the largest series are based on retrospective multicentre data collection and review. ${ }^{[3,4,9]}$

Owing to the frequently relatively benign presentation of the patient, many of these injuries are initially overlooked and presentation is therefore delayed, potentially causing severe morbidity and even mortality in some cases. ${ }^{[10]}$ The objectives of this survey and review were to determine how pancreatic trauma is managed at South African (SA) tertiary institutions compared with equivalent international centres, and to see if this is in keeping with current published guidelines on paediatric pancreatic trauma management.

\section{Methods}

A survey was emailed to 45 paediatric surgical consultants working in various paediatric surgical units in SA, Italy, England and Australia. The questionnaire comprised two scenarios of isolated pancreatic trauma (grade III), the main difference between the two scenarios being the time interval between initial injury and presentation. In the first scenario (A), the patient presented 6 hours post injury (Fig. 1) whereas in the second scenario (B), the patient presented 6 days post initial injury (Fig. 2). The survey, in a tick box format to facilitate analysis, enquired about diagnosis and subsequent work-up (including preferred imaging techniques), supportive management (including nutrition), the various options of definitive intervention and follow-up procedure.

\section{Results}

Twenty-one surgeons responded to the questionnaire from four countries consisting of 12 paediatric surgical units. In six of the units, more than one specialist returned the questionnaire.

\section{Scenario A}

Acute presentation of a ductal injury between the body and neck of the pancreas. Presentation was within 6 hours of incident, with no associated injuries (Fig. 1; Table 1). 


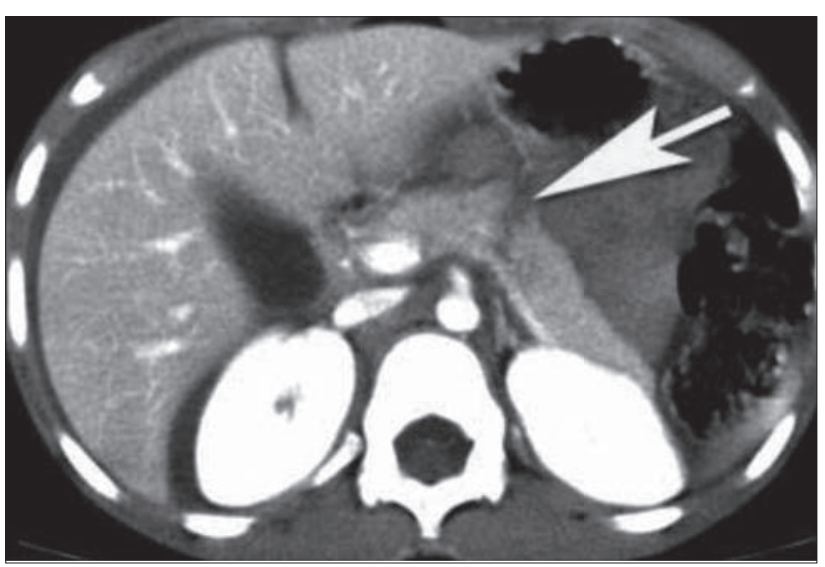

Fig. 1. Scenario A: Axial computed tomography abdomen with intravenous contrast demonstrating an isolated grade III pancreatic injury (arrow).

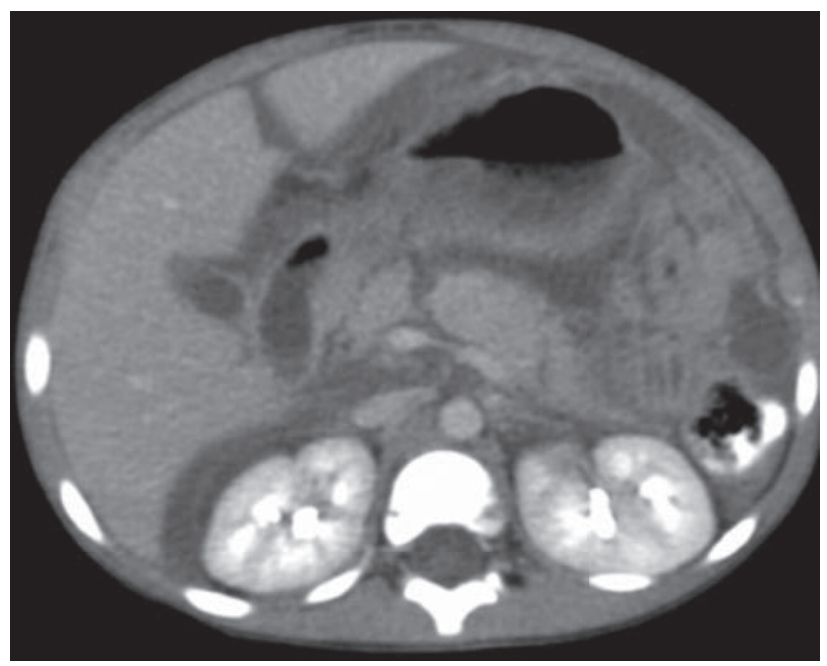

Fig. 2. Scenario B: Axial computed tomography abdomen with intravenous contrast demonstrating a delayed grade III isolated pancreatic injury.

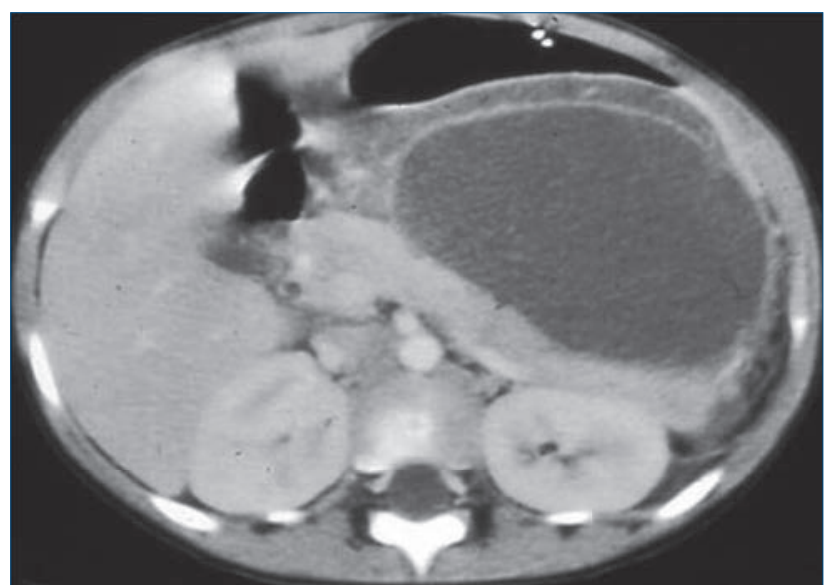

Fig. 3. Axial computed tomography abdomen with intravenous contrast demonstrating a pancreatic pseudocyst 6 weeks post injury.

Management of this injury varied between individuals and units, including individuals in the same unit. Of the 21 surgeons who responded:

1. Ten opted for surgical management:

- 5 for open distal spleen-sparing pancreatectomy

- 1 for open ductal repair

- 4 for laparoscopic distal spleen-sparing pancreatectomy.
2. A total of 8 opted for conservative management, namely pancreatic rest with total parenteral nutrition (TPN) until pain resolved, analgesia, and serial abdominal examinations. Of these, 7 would use octreotide to decrease pancreatic exocrine secretions.

If a pancreatic pseudocyst developed (Fig. 3; Table 2):

- 3 opted for open internal drainage

- 3 would do an endoscopic internal drainage procedure

- 2 would do percutaneous drainage.

3. Three opted for an ERCP and stent insertion.

\section{Scenario B}

Delayed presentation of a ductal injury between the body and neck of the pancreas. Presentation was 6 days post incident with no associated injuries (Fig. 2; Table 3).

1. Four surgeons would do an open distal spleen-sparing pancreatectomy despite the possibility of splenic injury.

2. A total of 13 opted for conservative management, of whom 9 would use octreotide.

If a pseudocyst developed:

- 7 opted for open internal drainage

- 4 would do an endoscopic internal drainage procedure

- 2 would do percutaneous drainage.

3. Four surgeons elected for an ERCP and transpapillary stent that would be left in situ:

- 2 would leave in situ for 1 month

- 1 would leave in situ for 2 months

- 1 would leave in situ for 3 months.

Nine would treat scenarios A and B in the same manner. Out of the 6 units that had multiple replies, 4 units had specialists with different management strategies.

Nutritional management during conservative management is summarised in Table 4. Fig. 4 graphically illustrates the difference in management choices between the two scenarios. Table 5 illustrates the differences between SA surgeons and their international colleagues.

\section{Discussion}

The severity of pancreatic trauma has been graded by the American Association for Surgery in Trauma (Table 6). The management of grade I and II injuries is less controversial, and conservative management is agreed as the standard of care. ${ }^{[1,3,7,8,10]}$ Treatment becomes less clear and more controversial when ductal injury is suspected (grades III - V). Some authors advocate conservative management irrespective of the grade of the injury, ${ }^{[1,5,6,11]}$ while others opt for an aggressive surgical approach if the diagnosis is made early on, preferring distal pancreatectomy with splenic preservation. ${ }^{[7,10,12,13]}$ This has been described via both laparotomy and laparoscopy. ${ }^{[13]}$ The alternative approach is stent insertion after diagnostic ERCP, in which the stent is inserted across the duct if possible, or alternatively into the peripancreatic fluid collection associated with the ductal defect. ${ }^{[8,14,15]}$ Other less commonly described techniques include creation of a Roux-en-Y jejunal onlay, primary ductal repair and drainage, oversew of proximal pancreatic stump and distal Roux-en-Y pancreaticojejunostomy. ${ }^{[16]}$ The results from our study similarly reflected this varied approach to the management of these complex injuries, with all three common management strategies being used.

The two scenarios were designed in response to numerous factors. Owing to the nonspecific nature of the signs and symptoms of isolated pancreatic trauma, it is uncommon for patients to present in the first 48 hours post injury, particularly in SA where access to healthcare is not always immediately available. ${ }^{[5]}$ Early presentation is more common 


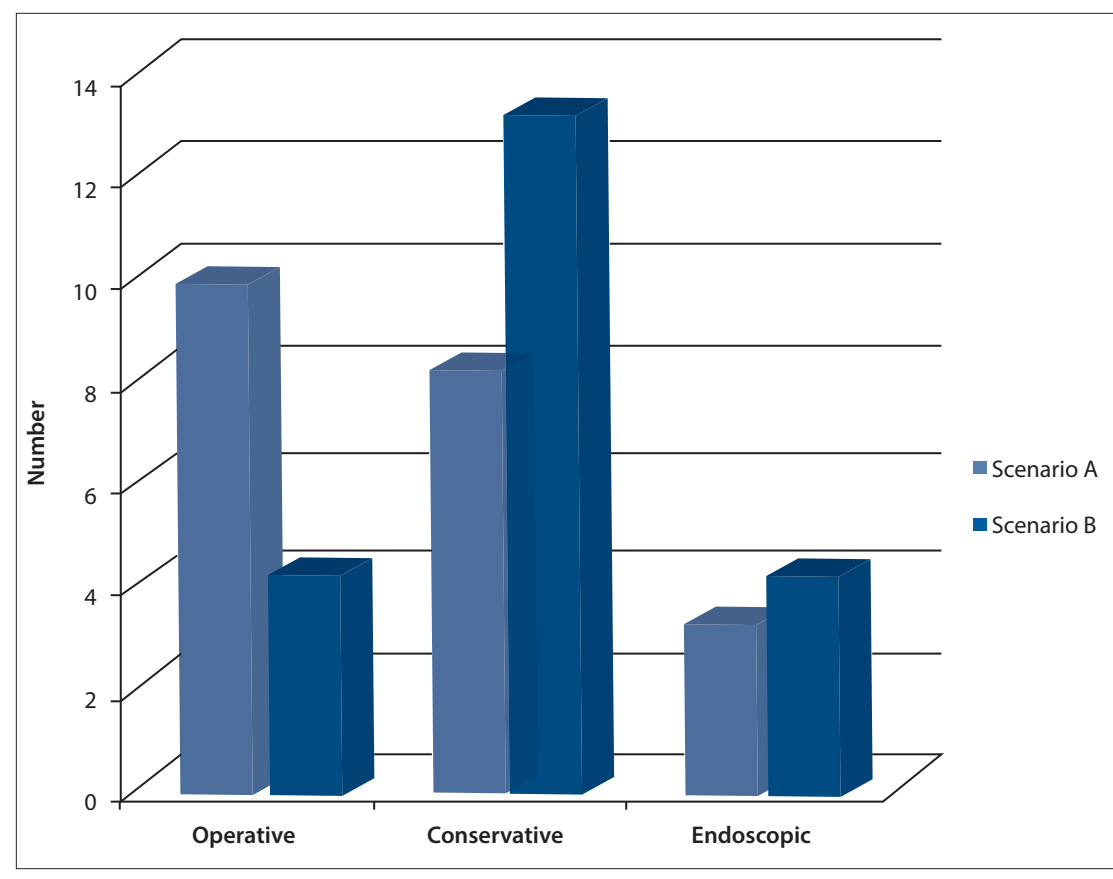

Fig. 4. Difference in management choices between scenarios $A$ and $B$.

Table 1. Management choices for Scenario A

\begin{tabular}{ll}
\hline Management & Specialists, $\boldsymbol{n}$ \\
\hline Operative & 10 \\
Conservative & 8 \\
ERCP with stent insertion & 3 \\
ERCP = endoscopic retrograde cholangiopancreatogram. &
\end{tabular}

Table 2. Management choices for a non-resolving pseudocyst (Scenario A)

\begin{tabular}{ll}
\hline Non-resolving pseudocyst management & Specialists, $\boldsymbol{n}$ \\
\hline Laparotomy with internal drainage & 3 \\
Endoscopically with internal drainage & 3 \\
Percutaneous drainage & 2
\end{tabular}

Table 3. Management choices for Scenario B

\begin{tabular}{ll}
\hline Management & Specialists, $\boldsymbol{n}$ \\
\hline Operative & 4 \\
Conservative & 13 \\
ERCP with stent insertion & 4 \\
ERCP = endoscopic retrograde cholangiopancreatogram. &
\end{tabular}

Table 4. Nutrition during conservative management

\begin{tabular}{ll}
\hline Nutrition during conservative management & Specialists, $\boldsymbol{n}$ \\
\hline NPO and IVI fluids until resolution & 0 \\
NPO and TPN until resolution & 3 \\
Naso-jejunal feeding & 1 \\
NPO and IVI fluids, then feed when pain resolved & 2 \\
NPO and TPN, then feed when pain resolves & 7 \\
NPO = nil per os; IVI = intravenous infusion; TPN = total parenteral nutrition. &
\end{tabular}

in developed countries, where cross-sectional imaging is more readily available, allowing earlier diagnosis of an injury and its extent. Once a significant period of time (24 - 48 hours) has elapsed after injury, it becomes far more difficult to perform a pancreatectomy without disrupting the vascular supply to the spleen. Since preservation of the spleen is important in children, the spleen should be spared if at all possible, hence the evolution of non-resective options for injuries that present in this time period.

There is considerable debate about the best imaging modality for blunt pancreatic trauma. Contrast computed tomography (CT) is considered the gold standard to diagnose pancreatic injuries, but its sensitivity and specificity change depending on the delay from the time of injury. In the first 12 - 24 hours post injury, pancreatic contusion or laceration can be missed owing to the evolving nature of the injury process. Furthermore, injuries can also be overlooked owing to the relative lack of visible retroperitoneal fat planes in children as well as the initial lack of reactive oedema. ${ }^{[8,17]}$ Canty and Weinman $^{[15]}$ demonstrated a $70 \%$ pick-up of pancreatic injury with initial contrast-enhanced CT scan $(n=11 / 16)$, whereas Houban et al. ${ }^{[8]}$ had a $91 \%(n=10 / 11)$ diagnostic rate with contrast CT. The improved accuracy in the second study could be due to the late referral of the cohort of patients, with the CT scans being performed more than 24 hours after injury. ${ }^{[8,18]}$ If the initial CT is negative and there is a high index of suspicion of a pancreatic injury, a repeat CT scan may need to be performed after 24 hours.

There is no specific role for ultrasound in diagnosing acute pancreatic injury; however, ultrasound is well established with respect to follow-up of previously documented injuries. ${ }^{[8]}$ The ease of performance, low cost and absence of radiation in ultrasound makes it ideal in monitoring the size and location of peripancreatic fluid collections and pseudocysts. Although the use of magnetic resonance cholangiopancreatography (MRCP) in paediatric pancreatic trauma has not been well studied, it is commonly used in centres all over the world. Houben et al. ${ }^{[8]}$ compared four MRCPs with ERCPs in their study and found excellent correlation in predicting the nature of the ductal injury. However, it should be noted that appropriate protocols are necessary to achieve adequate image quality and, even when adequate, MRCP lacks therapeutic ability, particularly when compared with ERCP. Nevertheless, in select cases and with adequate radiological expertise, it can be a useful non-invasive investigation that can guide management. More research is still needed to determine its absolute role.

Diagnostic ERCP is also controversial, with some authors believing that it should be 
Table 5. Difference in management choices between South African surgeons and international surgeons

\begin{tabular}{|c|c|c|}
\hline Management & South Africa $(N=15)$ & International $(N=6)$ \\
\hline & \multicolumn{2}{|c|}{$n(\%)$} \\
\hline \multicolumn{3}{|l|}{ Scenario A } \\
\hline Operative & $7(46.6)$ & $3(50.0)$ \\
\hline Conservative & $6(40.0)$ & $2(33.3)$ \\
\hline ERCP with stent & $2(13.3)$ & $1(16.6)$ \\
\hline \multicolumn{3}{|l|}{ Scenario B } \\
\hline Operative & $4(26.6)$ & $0(0.0)$ \\
\hline Conservative & $9(60.0)$ & $4(66.6)$ \\
\hline ERCP with stent & $2(13.3)$ & $2(33.3)$ \\
\hline
\end{tabular}

Table 6. American Association for Surgery in Trauma pancreatic trauma grading system

\begin{tabular}{ll}
\hline Grade & Pancreatic injury \\
\hline Grade I & $\begin{array}{l}\text { Haematoma with minor contusion/laceration but } \\
\text { without duct injury }\end{array}$ \\
Grade II & Major contusion/laceration but without duct injury \\
Grade III & Distal laceration or parenchymal injury with duct injury \\
Grade IV & $\begin{array}{l}\text { Proximal laceration or parenchymal injury with } \\
\text { injury to bile duct/ampulla }\end{array}$ \\
Grade V & Massive disruption to pancreatic head
\end{tabular}

undertaken in every patient with equivocal CT evidence of pancreatic trauma. ${ }^{[14,17,19]}$ Others feel that there is little benefit, considering the relatively high complication rates that include the possibility of introducing infection into a peripancreatic fluid collection, the development of post-ERCP pancreatitis and the conversion of a partial to complete ductal disruption. The risk of these complications has been estimated to be about $6 \%{ }^{[8]}$ If management options are to be directed by the extent of ductal involvement, it is probably justified to perform an ERCP, but if it is an academic exercise and it is not going to influence management, then the risks probably outweigh the benefits.

Serum amylase level is part of the blood investigation that is requested on any blunt abdominal trauma patient. There is no correlation between serum amylase level and severity of pancreatic injury, with the sensitivity of serum amylase to predict a pancreatic injury being $\sim 70 \%$. Jobst et al. ${ }^{[7]}$ found elevated admission amylase levels in 40 out of 56 children (71\%) with confirmed pancreatic injuries on imaging. Shilyansky et al. ${ }^{[5]}$ found hyperamylasaemia in 27 of $36(75 \%)$ of patients. It is important to repeat amylase levels if the clinical picture and radiology are suspicious of pancreatic trauma and the initial assay is normal, as the majority of amylase levels will be raised after the first 12 hours post injury. If there are associated facial injuries with suspicion that the raised amylase could possibly be coming from the salivary glands, then lipase levels can be checked. ${ }^{[17]}$

Once an injury has been documented and a conservative approach adopted, management comprises nasogastric tube placement, TPN, serial clinical examination and monitoring of amylase levels. This has been shown to be a safe approach to managing paediatric pancreatic trauma patients. ${ }^{[1,5,6,20,21]}$ It is accepted that there will be a higher incidence of pseudocyst formation, but proponents of this technique do not consider pseudocyst formation a complication. According to the latest Atlanta Classification for pancreatitis, a fluid collection around the pancreas in the first 4 weeks post injury is called an acute peripancreatic fluid collection. After 4 weeks, it is termed a pancreatic pseudocyst. Fifty to sixty per cent of acute peripancreatic fluid collections that develop secondary to pancreatic injury undergo spontaneous resolution within 6 weeks of injury. ${ }^{[12,21]}$ Those that persist and form pseudocysts that remain symptomatic, require some sort of definitive intervention. Cigdem et al. ${ }^{[20]}$ treated 25 paediatric pancreatic trauma patients conservatively; 11 pseudocysts developed, 5 resolved spontaneously and 6 required surgical intervention to drain the cyst. De Blaauw et al. ${ }^{[21]}$ treated 31 patients conservatively, with 6 having a grade III or IV injury; 14 developed pseudocysts but only 6 needed operative drainage. The majority of patients who are treated conservatively receive parenteral nutrition to rest the pancreas. This is weaned when abdominal pain resolves, amylase returns to normal levels and appetite returns. ${ }^{[1,5,20,21]}$ As can be seen in the results section from this survey, the majority of conservatively treated patients would have their parenteral nutrition stopped on resolution of pain.

Octreotide acetate infusion is used frequently in the conservative management of pancreatic trauma; its use is extrapolated from randomised trials in adults using octreotide in the treatment of pancreatic fistulas post pancreatic resection. Octreotide use results in reduced splanchnic blood flow and diminished pancreatic secretion of amylase, trypsin and lipase which intuitively should lead to enhanced recovery. ${ }^{[22]}$ However, there are currently no randomised, controlled trials proving that octreotide is effective in reducing complications of pancreatic trauma, although isolated case reports demonstrate that it may be effective. ${ }^{[23]}$

The advantage of conservative management is that the patient may avoid surgical intervention, on the basis that $50 \%$ of acute peripancreatic fluid collections may resolve spontaneously. In addition, the theoretical danger of decreased pancreatic endocrine and exocrine function later in life due to pancreatic resection may be averted. ${ }^{[6]}$

The technique of draining a non-resolving pseudocyst is also controversial. Most of the earlier literature describes percutaneous drainage. This seems to work in the majority of cases, but the risk of creating a pancreatic fistula is increased. ${ }^{[24]}$ Rescorla et al. ${ }^{[25]}$ describe failure of percutaneous drainage in three out of four patients when there is an associated ductal disruption. Stringer et al. ${ }^{[26]}$ describe five grade III injuries; all five developed pseudocysts. They were all drained percutaneously initially but failed to resolve, and all went on to definitive surgery. Millar et al. ${ }^{[27]}$ suggest percutaneous drainage only when the cyst is infected, while the remainder of non-resolving pseudocysts should have an open cystogastrostomy or Roux-en-Y internal drainage. The majority of recent papers discuss endoscopically placed stent-cystogastrostomy, transampullary transductal drainage or percutaneous drainage in the radiology suite. ${ }^{[8,9,28]}$ It would seem more logical to do an internal drainage technique if a true ductal injury is present, owing to the higher failure rate of percutaneous drainage. If these techniques do not resolve the pseudocyst, or if recurrence occurs, a more invasive open cystogastrostomy or Roux-en-Y is indicated.

Early surgical management of pancreatic trauma with ductal disruption (grade III) has also been given a lot of attention in the literature. It is usually performed in the first 48 hours after injury, minimising the risk of iatrogenic injury to the splenic vessels and need for splenectomy, which has an increased incidence thereafter. Proponents of early spleen-sparing pancreatectomy cite decreased length of hospital stay, decreased times on TPN, more rapid resumption of enteral feeds, lower morbidity and fewer repeat interventions as their reasons. ${ }^{[9,10]}$ Iqbal et al. ${ }^{[9]}$ compared 54 operated (spleen-sparing pancreatectomy) grade III injuries with 26 conservatively treated grade III injuries. They had no pseudocyst development in the operated group, but $44 \%$ of the conservatively managed group developed 


\section{RESEARCH}

pseudocysts. The overall morbidity, time to initial feeds, time to full feeds, total days in hospital and time to complete resolution were all significantly reduced in the operated group. However, performing a spleen-sparing pancreatectomy does have some risk. Pancreatic leak does occur, documented as $7 \%$ by Iqbal et al. Meier et al. ${ }^{[10]}$ operated on nine patients, with morbidities including one pancreatic leak, one prolonged ileus and one postoperative bowel obstruction. In another study, a pseudocyst developed in $21 \%$ of the operated group, suggesting pancreatic leak that required surgical intervention. ${ }^{[19]}$ The literature reports a complication rate of between $20 \%$ and $35 \%$ for early spleensparing pancreatectomy.

The concern about performing a pancreatic resection is that there are no long-term studies documenting the impact on long-term endocrine or exocrine function. It is known that a $65 \%$ loss of $\beta$-cells in adults causes impairment of glucose tolerance. It is therefore theoretically possible that children with substantial pancreatic loss could be at risk of endocrine deficiency later in life. ${ }^{[29]}$ However, it has been reported that in conservatively managed patients with grade III injuries, two-thirds of the patients' distal pancreas underwent atrophy, with recanalisation of the remaining third. ${ }^{[6]}$ There has been one case report of a genetically susceptible child who developed diabetes mellitus 3 years after a blunt pancreatic injury. Long-term follow-up on all pancreatic injuries is therefore suggested. ${ }^{[29]}$

As paediatric gastroenterologists are gaining more experience in performing ERCPs on children, endoscopic techniques are becoming more popular in the management of these patients. The concerns regarding ERCP on children are related to the technical difficulty of cannulating a smaller ampulla, and its possible complications as discussed earlier. There is also the problem of migrating stents requiring multiple reinsertions and the possibility of stricture formation after stent removal at the site of injury. ${ }^{[20]}$ The advantage of an ERCP is that it is diagnostic in identifying ductal injury and therapeutic when stents are inserted. Another advantage is that there is no time constraint in management of trauma patients. An ERCP can be done on day 1 or day 6 post injury when necessary. Canty and Weinman ${ }^{[15]}$ were the first to report the use of ERCP and stent insertion in their two-patient case report in 2001. Houben et al. ${ }^{[8}$ reported on 12 patients who underwent ERCP at a mean time of 11 days post injury. Nine of those patients managed to have a transductal endoscopic stent inserted, and there were two technical failures. The remaining patient had an intact duct on ERCP. Three of the nine successful stents had to be replaced with a bigger stent for improved drainage. Minor complications occurred in some of the patients: 5 had a transient rise in their amylase, 2 had increased epigastric pain for 48 hours post procedure, 4 had to have percutaneous aspiration/ drainage of pseudocysts and 2 required cystgastrostomy. Therefore, despite the placement of an endoscopic stent, $50 \%$ of the patients went on to develop a pseudocyst that required a drainage procedure of some type. These outcomes may be skewed by the fact that the series reported comes from a referral institution, which only sees complicated cases. Unfortunately, paediatric ERCP is not available in many centres, but has a well-documented role in the management of paediatric pancreatic trauma, including diagnosis and treatment in the acute setting, as well as management of established pseudocysts in the long term.

SA paediatric surgeons' management of pancreatic injury with ductal involvement mimics that reported in the literature. The only real difference in management is that the operative approach in the international cohort is more likely to be laparoscopic. This could be due to a lower theatre burden and more cosmetically sensitive population group in the First World. One pitfall of this type of review is that financial incentives pertaining to the different management techniques are not accounted for when reviewing the decisions made by treating physicians.

\section{Conclusion}

There is still no clear ideal management of grade III paediatric pancreatic injuries. It is accepted that grade I and II patients should be treated conservatively. If a pseudocyst develops and becomes symptomatic or does not resolve spontaneously, there are a multitude of ways to drain it either internally or externally. The controversy arises in grade III injuries. SA paediatric surgeons, much like their overseas counterparts, follow the international published guidelines with a vast array of management protocols as a result. From both the literature and the results of the survey, there seems to be no ideal way of managing these patients when there is an associated ductal injury. Proponents of each technique (operative, conservative or endoscopic) claim that their technique is superior and safer than the other. Fortunately, all techniques are effective, with a relatively low morbidity. Mortality is usually due to associated injuries. No absolute algorithm can be used to treat these patients. All patients should be treated individually and managed with an approach and techniques that are feasible in keeping with facilities available in each individual unit. Factors that should be taken into account include associated injuries, length of time since injury, facilities available, grade of injury and capability of the paediatric surgical team.

\section{References}

1. Keller MS, Stafford PW, Vane DW. Conservative management of pancreatic trauma in children. J Trauma 1997;42(6):1097-1100

2. Jacombs AS, Wines M, Holland AJA, Ross FI, Shun A, Cass DT. Pancreatic trauma in children. Pediatr Surg 2004;39:96-99.

3. Paul MD, Mooney DP. The management of pancreatic injuries in children: Operate or observe. Pediatr Surg 2011;46(6);1140-1143. [http://dx.doi.org/10.1016/j.jpedsurg.2011.03.041]

4. Mattix KD, Tataria M, Holmes I, et al. Pediatric pancreatic trauma: Predictors of nonoperative management failure 4. Mattix KD, Tataria M, Holmes], et a. Pediatric pancreatic trauma: Predictors of nonoperative management failu and assoch To 5. Shilyansky J, Sena LM, Kreller M, et al. Nonoperative management of pancreatic injuries in children.

6. Wales PW, Shuckett B, Kim PCW. Long-term outcome after nonoperative management of complete traumatic pancreatic transection in children. J Pediatr Surg 2001;36(5):823-827. [http://dx.do org $/ 10.1053 /$ jpsu.2001.22970

7 Jobst MA, Canty TG Sr, Lynch FP. Management of pancreatic injury in pediatric blunt abdominal trauma. J Pediatr Surg 1999;34(5):818-824.

8. Houben $\mathrm{CH}$, Ade-Ajayi N, Patel S, et al. Traumatic pancreatic duct injury in children: Minimally invasive approach to management. J Pediatr Surg 2007;42(4):629-635. [http://dx.doi.org/10.1016/j.jpedsurg.2006.12.025] 9. Iqbal CW, St Peter SD, Tsao K, et al. Operative vs nonoperative management for blunt pancreatic transection in children: Multi-institutional outcomes. J Am Coll Surg 2014;218(2):157-162. [http:// dx.doi.org/10.1016/j.jam collsurg.2013.10.012]

10. Meier DE, Coln CD, Hicks BA, Guzzetta PC. Early operation in children with pancreas transection. J Pediatr Surg 2001;36(2):341-344. [http://dx.doi.org/10.1053/jpsu.2001.20711]

1. Kouchi K, Tanabe M, Yoshida H, et al. Nonoperative management of blunt pancreatic injury in childhood. J Pediatr Surg 1999;34(11):1736-1739. [http://dx.doi.org/10.1016/S0022-3468(99)90657-7]

12. Beres AL, Wales PW, Christison-Lagay ER, McClure ME, Fallat ME, Brindle ME. Non-operative management of high-grade pancreatic trauma: Is it worth the wait? J Pediatr Surg 2013;48(5):10601064. [http://dx.doi.org/10.1016/j.jpedsurg.2013.02.027]

13. Iqbal CW, Levy SM, Tsao K, et al. Laparoscopic versus open distal pancreatectomy in the managemen of traumatic pancreatic disruption. J Laparoendosc Adv Surg Tech A 2012;22(6):595-598. [http:// dx.doi.org/10.1089/lap.2012.0002]

14. Rescorla FJ, Plumley DA, Sherman S, Scherer LR 3rd, West KW, Grosfeld JL. The efficacy of early ERCP in pediatric pancreatic trauma. J Pediatr Surg 1995;30(2):336-340.

15. Canty TG, Weinman D. Treatment of pancreatic duct disruption in children by an endoscopically placed stent. J Pediatr Surg 2001;36(2):345-348. [http://dx.doi.org/10.1053/jpsu.2001.20712]

16. Stringer MD. Pancreatitis and pancreatic trauma. Semin Pediatr Surg 2005;14(4):239-246. [http:// dx.doi.org/10.1053/j.sempedsurg.2005.06.007]

17. Maeda K, Ono S, Baba K, Kawahara I. Management of blunt pancreatic trauma in children. Pediatr Surg Int 2013;29:1019-1022. [http://dx.doi.org/10.1007/s00383-013-3402-9]

8. Canty TG Sr, Weinman D. Management of major pancreatic duct injuries in children. J Trauma 2001;50(6):1001-1007

19. Wood JH, Partrick DA, Bruny LL, Sauaia A, Moulton SL. Operative vs nonoperative management of blunt pancreatic trauma in children. J Pediatr Surg 2010;45(2):401-406. [http://dx.doi.org/10.1016/j.jpedsurg.2009.10.095]

20. Cigdem MK, Senturk S, Onen A, Siga M, Akay H, Otcu S. Nonoperative management of pancreatic injuries in pediatric patients. Surg Today 2011;41(5):655-659. [http://dx.doi.org/10.1007/s00595-010-4339-4]

1. De Blaauw I, Winkelhorst JT, Rieu PN, et al. Pancreatic injury in children: Good outcome of nonoperative treatment. J Pediatr Surg 2008;43(9):1640-1643. [http://dx.doi.org/10.1016/j.jpedsurg.2008.03.061]

2. Nwariaku FE, Terracina A, Mileski WJ, Minei JP, Carrico CJ. Is octreotide beneficial following pancreatic injury? Am J Surg 1995; 170(6):582-585.

23. Wensil AM, Balasubramanian SA, Bell TL. Resolution of a posttraumatic pancreatic pseudocyst with octreotide acetate in a pediatric patient. Pharmacother J Pharmacotherapy 2011;31(9):924. [http:// dx.doi.org/10.1592/phco.31.9.924]

24. Bass J, Lorenzo MD, Desjardins JG, Grignon A, Ouimet A. Blunt pancreatic injuries in children: The role of percutaneous external drainage in the treatment of pancreatic pseudocysts. J Pediatr Surg 1988:23(8):721-724. 5. Rescorla FJ, Cory D, Vane DW, Wes KW, Grosfeld JL. Failure of percutaneous drainage in children with traumatic pancreatic pseudocysts. J Pediatr Surg 1990;25(10):1038-1042.

26. Stringer MD. Pancreatic trauma in children. Br J Surg 2005;92(4):467-470. [http ///dxdoi.org/10.1002/bjs.4876] 27. Millar AJW, Rode H, Stunden RJ, Cywes S. Management of pancreatic pseudocysts in children Pediatr Surg 1988;23(2):122-127.

28. Abbo $\mathrm{O}$, Lemandat $\mathrm{A}$, Reina $\mathrm{N}$, et al. Conservative management of blunt pancreatic traum in children: A single center experience. Eur J Pediatr Surg 2013:23(6):470-473. [http://dxdol. org/10.1055/s-0033-1333642]

29. Edwards MI, Crudo DF, Carlson TL, Pedersen AM, Keller L. Pancreatic atrophy and diabetes mellitus following blunt abdominal trauma. J Pediatr Surg 2013:48(2):432-435. [http://dx.doiorg/10.1016/. jpedsurg.2012.11.030 